\title{
Deconfinement, Chiral Symmetry Breaking and Chiral Polarization
}

\section{Andrei Alexandru}

The George Washington University, Washington, DC, USA

E-mail: aalexanegwu.edu

\section{Ivan Horváth ${ }^{* \dagger}$}

University of Kentucky, Lexington, KY, USA

E-mail: horvath@pa.uky.edu

We examine the feasibility of the proposition that there is a temperature range $\mathrm{T}_{c}<\mathrm{T}<\mathrm{T}_{c h}$ in $\mathrm{N}_{f}=0$ QCD, where real Polyakov line (deconfined) vacuum exhibits valence spontaneous chiral symetry breaking and dynamical chiral polarization of Dirac eigenmodes. Detailed finite-volume analysis convincingly demonstrates the existence of such phase at fixed cutoff $(a=0.085 \mathrm{fm})$. Moreover, it is found that this behavior also takes place closer to the continuum limit $(\mathrm{a}=0.060$ fm) without qualitative change in its properties.

The 32nd International Symposium on Lattice Field Theory,

23-28 June, 2014

Columbia University New York, $N Y$

\footnotetext{
* Speaker.

${ }^{\dagger}$ We are indebted to Mingyang Sun for providing some of the illustrative graphics.
} 
1. The Context. This presentation is concerned with two proposed features in SU(3) gauge theories that closely relate to Dirac eigenmodes. The first one suggests [1] that, when $\mathrm{N}_{f}=0$ system deconfines into "real Polyakov line" vacuum at $T_{c}$, valence chiral symmetry remains broken for some range of temperatures $\mathrm{T}_{c}<\mathrm{T}<\mathrm{T}_{c h}$. Only after crossing such anomalous phase does the system become both deconfined and chirally symmetric.

The second feature proposes that $\mathrm{SU}(3)$ gauge theory that breaks valence chiral symmetry supports a layer of chirally polarized Dirac modes at low end of the spectrum [2,3], as shown schematically in Fig. 1 (left). Such layer is only well-defined via dynamical polarization methods [4], and its existence is indicated e.g. by volume density $\Omega$ of participating modes. The above relationship is conjectured to be an equivalence: splitting $\mathrm{SU}(3)$ theories into chirally broken and chirally symmetric is to yield the same result as partition into chirally polarized and chirally anti-polarized cases (see Fig. 1(right) for details). Note that $\eta$ is a massless valence field and the question of symmetry breakdown is well-defined with dynamical quarks of arbitrary masses.

The above proposals are each interesting in their own right. Indeed, the real Polyakov line vacuum of deconfined $\mathrm{N}_{f}=0$ theory is a good qualitative proxy to gauge vacuum of "real world" QCD above finite-temperature transition. It is thus important to demonstrate that the anomalous phase is a true feature of $\mathrm{N}_{f}=0$ theory, i.e. that of infinite-volume continuum system. In the other case, the validity of chiral polarization connection significantly constrains possible mechanisms of spontaneous chiral symmetry breaking for SU(3) theories in a model-independent way. After all, chiral condensate and chiral polarization are not required to be tied together by general principles.

However, the main reason we brought the above two aspects together here is that the former provides for a particularly sensitive test of the latter: is valence spontaneous chiral symmetry breaking (vSChSB) - chiral polarization (ChP) correspondence satisfied in corners of $\mathrm{SU}(3)$ theory space that are in anomalous phase?

2. The Tools. To detect vSChSB we use the fact that this phenomenon is equivalent to Dirac mode condensation. As such, it is indicated by non-zero density of near-zero modes $\rho(\lambda \rightarrow 0)$ in the infinite volume limit. The presence of chiral polarization will be signaled by volume density $\Omega$ of polarized modes, on whose definition we now briefly elaborate.
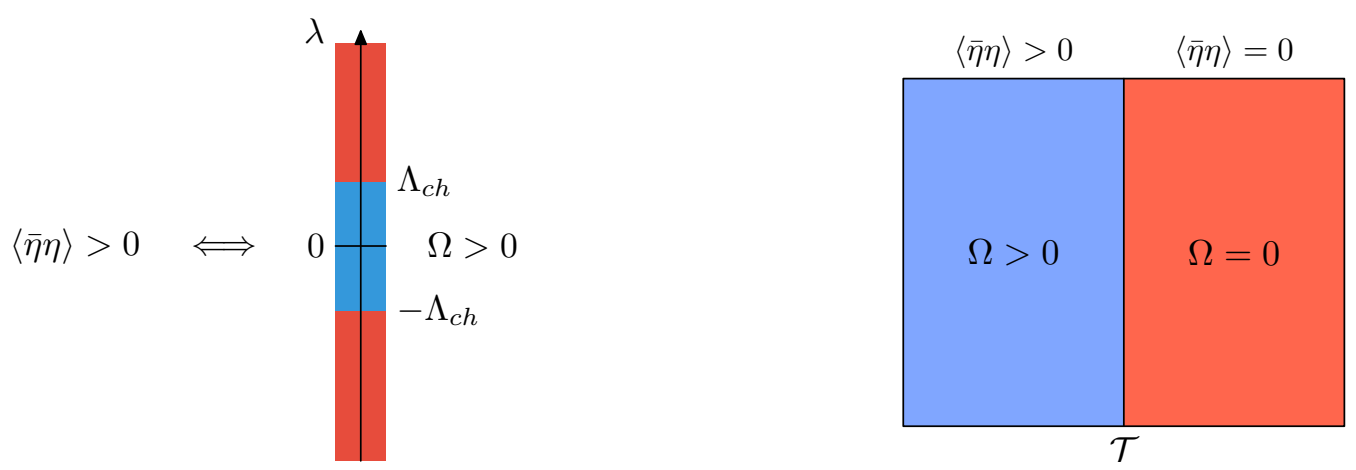

Figure 1: Schematic representation of vSChSB-ChP correspondence: $\eta$ denotes massless valence field, $\Omega$ volume density of chirally polarized modes, and $\Lambda_{c h}$ chiral polarization scale. Set $\mathcal{T}$ consists of SU(3) gauge theories with $\mathrm{N}_{f}$ arbitrarily massive fundamental fermions at arbitrary temperature. 
The concept of dynamical ChP is based on a correlation coefficient $C_{A}$ of Ref. [4], uniquely associated with property of polarization. Positive correlation implies tendency for asymmetry in left and right (chiral polarization), while the negative correlation signifies tendency for symmetry (chiral anti-polarization). Since $C_{A}$ can be assigned to every mode, it is useful to define the associated spectral constructs $[2,3]$. For example, cumulative polarization density $\sigma_{c h}(\lambda)$ is considered in addition to the usual cumulative mode density $\sigma(\lambda)$. In particular

$$
\sigma_{c h}(\lambda, V) \equiv \frac{1}{V}\left\langle\sum_{0<\lambda_{k}<\lambda} C_{A, k}\right\rangle \quad \sigma(\lambda, V) \equiv \frac{1}{V}\left\langle\sum_{0<\lambda_{k}<\lambda} 1\right\rangle
$$

where $\langle\ldots\rangle$ denotes the ensemble average. Exact zero modes are not included since their contribution vanishes in thermodynamic limit. In chirally polarized theory, $\sigma_{c h}(\lambda)$ will grow away from zero until it hits the edge of chirally polarized layer $\Lambda_{c h}$. The value of $\sigma$ at this point is $\Omega$.

It is convenient to eliminate $\lambda$ from the above relationships and consider $\sigma_{c h}=\sigma_{c h}(\sigma)$ [3]. Here too, chiral polarization reveals itself as a positive bump in the vicinity of the origin, defining $\Omega$ and volume density of total chirality $\Omega_{c h}$ simultaneously (see Fig. 2). If $\sigma_{c h}(\sigma)$ turns negative away from $\sigma=0$, or remains zero, there is no chirally polarized layer and $\mathrm{vSChSB}-\mathrm{ChP}$ correspondence predicts that valence chiral symmetry will not be broken in the infinite-volume limit.

All results presented here relate to Wilson gauge action and scale set via $r_{0}=0.5 \mathrm{fm}$. We use overlap Dirac operator [5] ( $\rho=26 / 19)$ with Wilson-Dirac kernel $(r=1)$ as a massless valence probe. As emphasized already, our interest is in deconfined vacuum with real Polyakov line.

3. Qualitative Overview. To start this inquiry, we seek to reproduce the qualitative observation in Ref. [1] of anomalous accumulation of overlap near-zeromodes past deconfinement temperature $T_{c}$, and to check whether their chiral polarization properties concur with vSChSB-ChP correspondence. Using the fixed scale approach on $20^{3} \times \mathrm{N}_{t}$ lattice, we change temperature across $T_{c}$. With scale set at $a=0.085 \mathrm{fm}(\beta=6.054)$, the fixed 3 -volume is $(1.7 \mathrm{fm})^{3}$ and the nominal continuumextrapolated deconfinement transition (see Ref. [6]) occurs just above $\mathrm{N}_{t}=8$.

While detailed account of these calculations covering wide range of temperatures is given elsewhere [2,3], Fig. 3 shows the relevant overview results for $\mathrm{N}_{t}=8,7,6$. Scatter plots of the
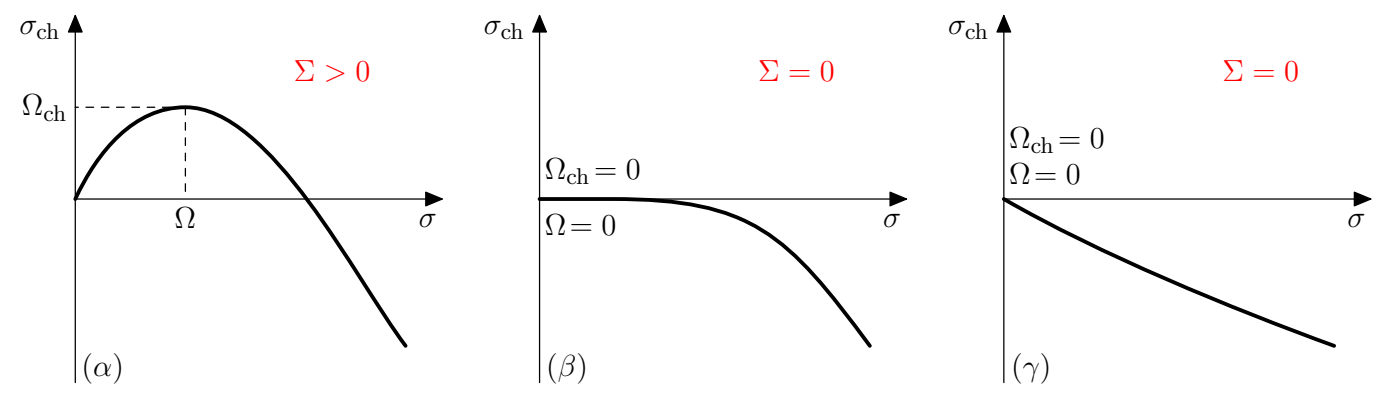

Figure 2: Behaviors of $\sigma_{c h}(\sigma)$ in the vicinity of $\sigma=0$. vSChSB-ChP correspondence associates case $(\alpha)$ with spontaneously broken valence chiral symmetry while cases $(\beta)$ and $(\gamma)$ with symmetric vacuum. 

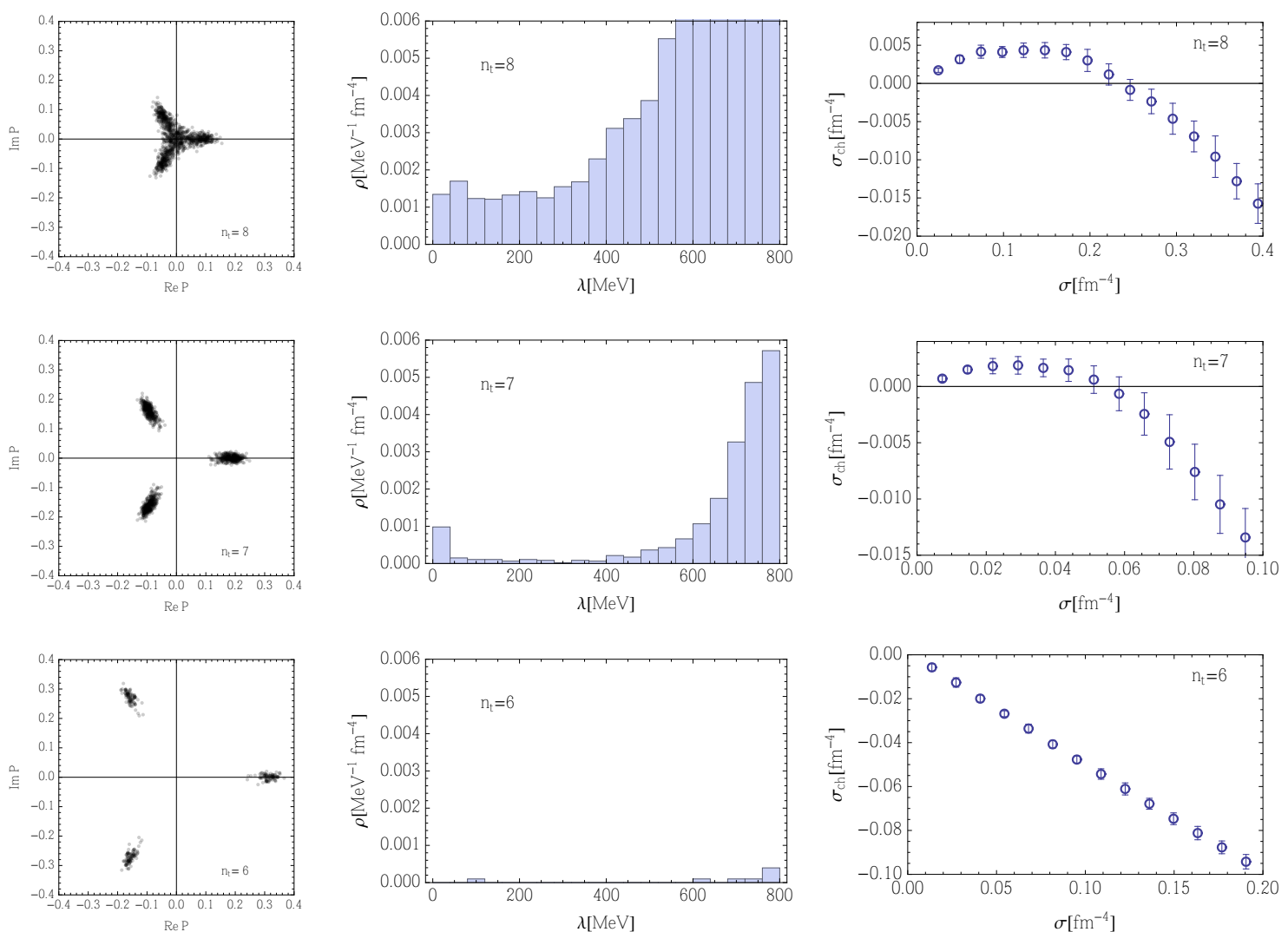

Figure 3: Indicators of deconfinement (left column), valence chiral symmetry breaking (middle column) and chiral polarization (right column) on $20^{3} \times \mathrm{N}_{t}$ lattices. See discussion in the text.

Polyakov line in left column confirm that $\mathrm{N}_{t}=8$ system behaves as borderline-deconfined, while at $\mathrm{N}_{t}=7$ and 6 it is manifestly deconfined. Nevertheless, the mode density data in the second column shows abundant near-zeromodes at $\mathrm{N}_{t}=7$ of anomalous type (non-monotonic $\rho(\lambda)$ ) observed in [1]. These modes disappear at $\mathrm{N}_{t}=6$, suggesting valence chiral transition between $\mathrm{N}_{t}=7$ and 6 . In the third column of Fig. 3, the behavior of $\sigma_{c h}(\sigma)$ clearly indicates the onset of chiral anti-polarization at the same place, in accordance with vSChSB-ChP correspondence.

3. Fixed Cutoff. Since symmetry breaking is involved in both features studied here, we are dealing with infinite-volume propositions, and finite-volume considerations are important. In particular, it is essential to truly establish the existence of deconfined chirally broken dynamics on the lattice, i.e. to carefully study volume trends at fixed cutoff [3]. One explicit worry is that the observed anomalous behavior could be an unwanted feature of the overlap construction that may be "cured" by large volumes in a way similar to the exceptional configuration issue.

To examine this, we study the $\mathrm{N}_{t}=7$ theory which clearly offers itself as a candidate of dynamics in anomalous phase. Thus, $\mathrm{N}^{3} \times 7$ systems are considered at the previously mentioned gauge coupling, and $\mathrm{N}=16,20,24,32$. The largest volume in the sequence is thus $(2.72 \mathrm{fm})^{3}$. The closeup of its spectral density at low end of the spectrum is provided in Fig. 4 (left), clearly confirming the anomalous accumulation of near-zero modes. To check volume tendencies, we consider a coarsegrained mode condensation parameter $\rho(\lambda=0, \Delta, V) \equiv \sigma(\lambda=\Delta, V) / \Delta$. Infinite-volume limit at 

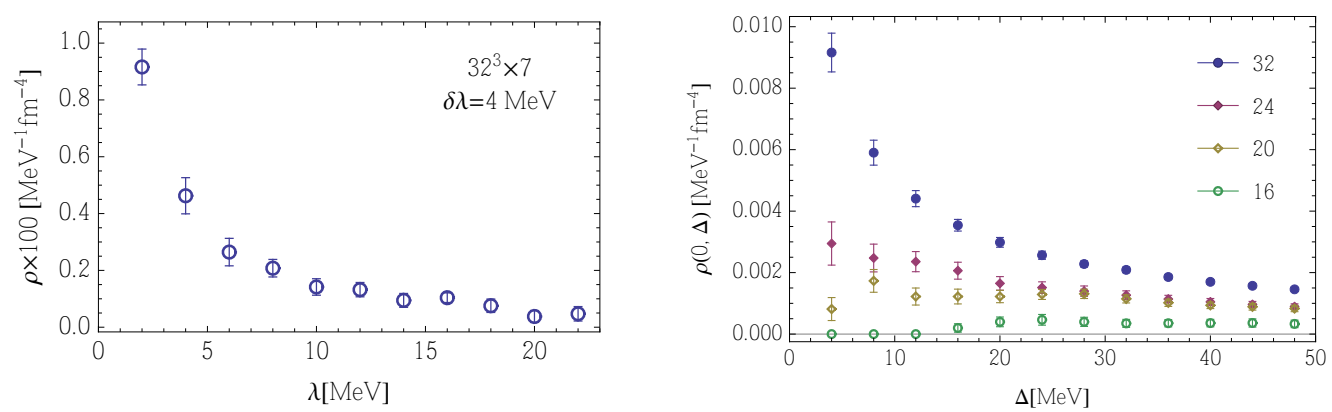

Figure 4: Spectral mode density in the vicinity of origin at $\mathrm{N}_{t}=7$ for the largest volume (left) and the mode (chiral) condensation parameter as a function of coarse-graining range $\Delta$ (right).

fixed coarse-graining parameter $\Delta$ needs to be taken before $\Delta \rightarrow 0$ defines the condensate. Fig. 4 (right) shows $\rho(0, \Delta)$ for above volumes down to $\Delta=4 \mathrm{MeV}$. Essential feature of these results is that $\rho(0, \Delta, V)$ grows as a function of volume at arbitrary accessible $\Delta$ : larger volumes enhance the density of near-zero modes. Moreover, the rate of increase grows with decreasing $\Delta$ making it extremely unlikely that the mode (chiral) condensate vanishes, and confirming that $\rho(\lambda)$ exhibits anomalous (monotonically decreasing) behavior near the origin in the infinite volume limit.

Given the above result, if vSChSB-ChP correspondence is obeyed by the regularized system in question, chiral polarization has to be present at least in arbitrarily large finite volume (see Conjecture 3 of Ref. [3]). To check for this, we plot $\sigma_{c h}(\sigma)$ for different volumes in Fig. 5. As one can see, not only is the system chirally polarized in each casee, the degree of chiral polarization increases as the system grows. For example, the density of chirally polarized modes $\Omega$ (position of the maximum in $\left.\sigma_{c h}(\sigma)\right)$ monotonically increases with volume [3].
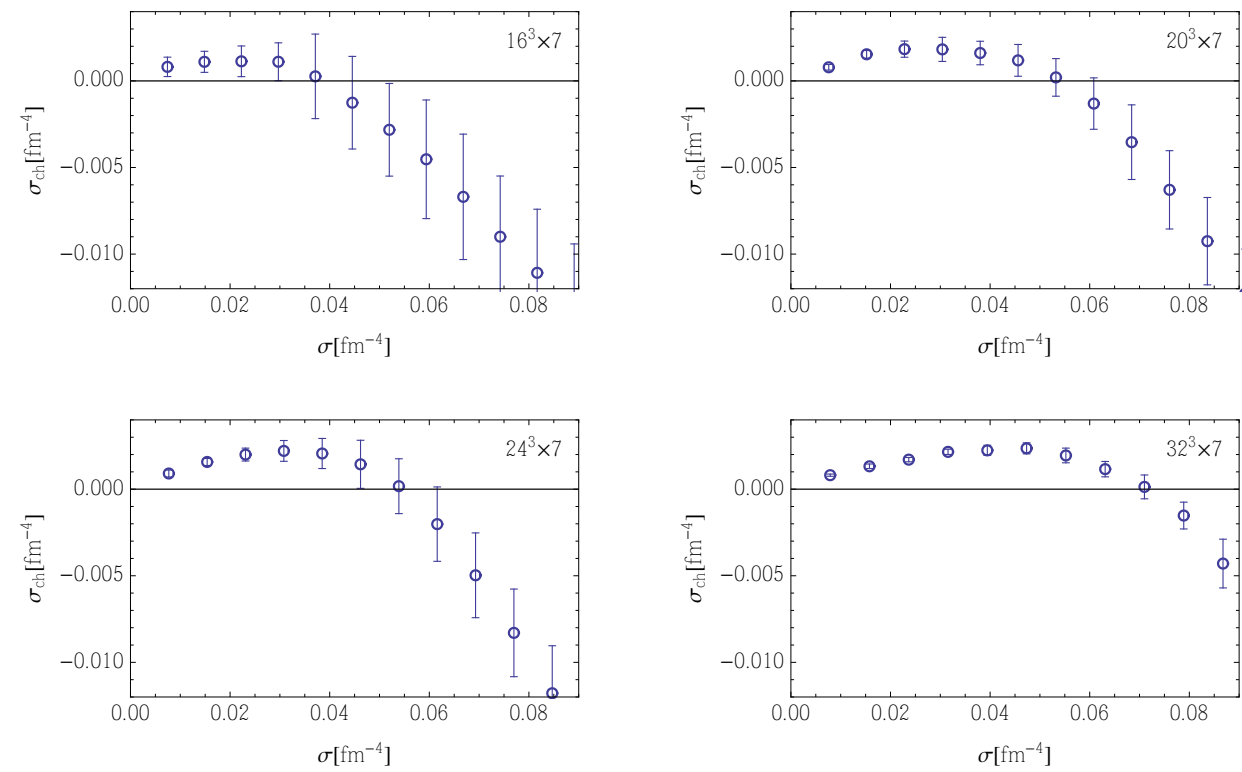

Figure 5: Behavior of $\sigma_{c h}(\sigma)$ at $\mathrm{N}_{t}=7$ : degree of chiral polarization increases with increasing volume. 
4. Continuum Limit. Another very relevant concern regarding the reality of chirally broken deconfined phase is that the observed anomalous behavior might be due to lattice artifacts that will disappear sufficiently close to the continuum limit. To examine this possibility, we consider the same system at significantly larger cutoff, where "the same" refers to identical $T / T_{c}$. Since $r_{0}$ serves to determine the scale, it is convenient to use the universal continuum-extrapolated result $r_{0} T_{c}=0.7498(50)$ of Ref. [7] to fix $T / T_{c}$. The systems of the previous section were at $a / r_{0}=$ 0.1701 , which for $N_{t}=7$ translates into $T / T_{c}=1.12$. We now switch to $a / r_{0}=0.1190$ and $N_{t}=10$ leading to the same $T / T_{c}$ but with lattice spacing lowered to $a=0.0595 \mathrm{fm}$.

Using the above setup we study the $34^{3} \times 10$ lattice which entails the same spatial volume as the $24^{3} \times 7$ system of the previous section $(\mathrm{L}=2.0 \mathrm{fm})$ to facilitate the most direct comparison. In Fig. 6 (top) we show spectral density $\rho(\lambda)$ at low end of the spectrum for the two cases. As one can see, the anomalous behavior is clearly present at higher cutoff as well. While there is some reduction in the width of the peak, there is no observable change in its height, i.e. the density in the vicinity of the origin scales within the available statistics (400 configurations).

Moreover, the plots of $\sigma_{c h}(\sigma)$ at the bottom of Fig. 6 reveal that the theory also remains chirally polarized. Combined with the type of volume scaling observed in the previous section, this leads us to conclude that the existence of anomalous phase and vSChSB-ChP correspondence both hold on the fine lattice. Also, while some decrease in $\Omega$ can be observed at larger cutoff, this is mainly related to the aforementioned reduction in the width of the anomalous peak rather than to changing polarization properties of the relevant modes as continuum limit is approached.
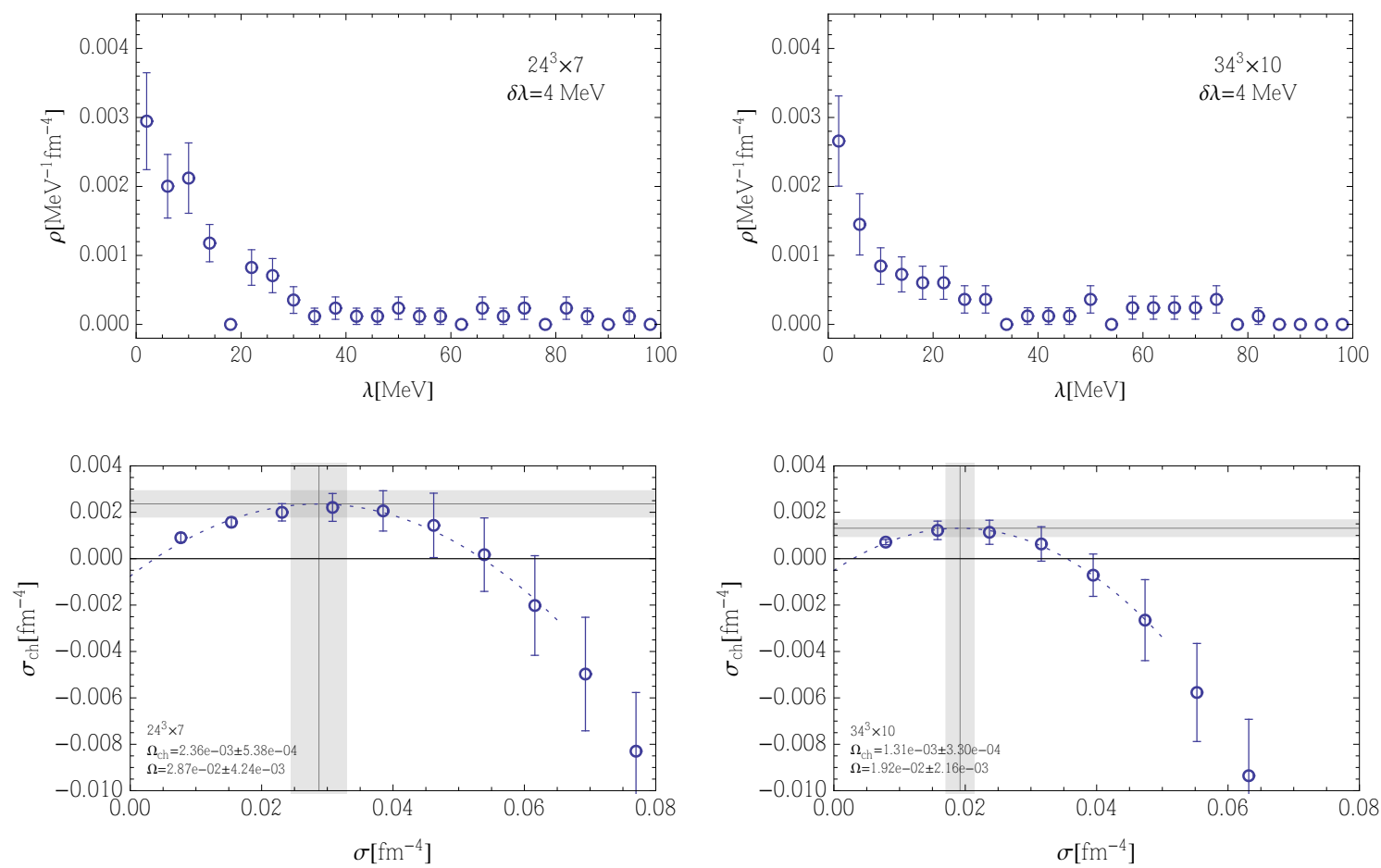

Figure 6: Comparison of spectral density close to zero (top) and cumulative chiral polarization $\sigma_{c h}(\sigma)$ (bottom) for systems at $T / T_{c}=1.12$, with the same volume ( $\left.\mathrm{L}=2 \mathrm{fm}\right)$, but with $a=0.0850 \mathrm{fm}$ (left) and $a=0.0595 \mathrm{fm}$ (right). 
5. Discussion. Resolving the existence of anomalous phase $T_{c}<T<T_{c h}$ in $\mathrm{N}_{f}=0$ QCD is important for understanding the nature of QCD transition region. While only an approximation to the "real world", the long history of quenched simulations shows that the system retains most qualitative features of full QCD, especially those related to gauge fluctuations. This applies to vSChSB which probes correlations of the gauge vacuum leading to symmetry breakdown in external fields interacting with it [3]. Thus, if ascertained to exist for $\mathrm{N}_{f}=0$ in real Polyakov line vacuum, the occurrence of anomalous phase in QCD with dynamical quarks would not be surprising. Some indications of such behavior have in fact been reported (see e.g. Refs. [8, 9, 10]).

However, the whole scenario could well be just a distortion due to infrared cutoff being too high or ultraviolet cutoff being too small. One warning is that the anomalous behavior is only apparent with overlap-type fermions. While one naturally tends to assume that chiral formulation is more reliable, it is also possible that overlap is "too sensitive" to chirality and topology in this case, and simply displays artifacts that other formulations cannot see. Thus, it is the reality of deconfined and chirally broken phase that is our main focus here and, due to the non-trivial nature of involved extrapolations, this can be most realistically settled in $\mathrm{N}_{f}=0$ theory.

We presented some of the data, first published in Ref. [3], that convincingly show stability of the phenomenon in the infrared: there indeed exists lattice $\mathrm{N}_{f}=0$ theory that is simultaneously deconfined and chirally broken. In addition, we analyzed previously unpublished data indicating that a substantial increase in lattice cutoff doesn't change the situation appreciably. Thus, while additional cutoff levels will be examined to make final conclusions, data available at present is consistent with the phase persisting to the continuum limit.

In the same vein, the data presented here supports the validity of vSChSB-ChP correspondence for physics in the anomalous phase. Since the nature of underlying dynamics changes substantially across deconfinement transition, this is one of the crucial tests for the emerging association between chiral symmetry breaking and chiral polarization.

\section{References}

[1] R.G. Edwards, U.M. Heller, J.E. Kiskis, R. Narayanan, Phys. Rev. D61, 074504 (2000), arxiv:hep-lat/9910041.

[2] A. Alexandru, I. Horváth, Phys. Lett. B722, 160 (2013). arXiv: 1210 . 7849.

[3] A. Alexandru, I. Horváth, arXiv:1405.2968.

[4] A. Alexandru, T. Draper, I. Horváth, T. Streuer, Annals Phys. 326, 1941 (2011), arXiv:1009.4451.

[5] H. Neuberger, Phys. Lett. B417 (1998) 141; Phys. Lett. B427 (1998) 353.

[6] F. Karsch, Nucl. Phys. Proc. Suppl. 60A, 169 (1998), arXiv: hep-lat/9706006.

[7] S. Necco, Nucl. Phys. B683, 137 (2004). arXiv: hep-lat/ 0309017

[8] M. Buchoff et al., Phys. Rev. D89, 054514 (2014), arXiv: 1309.4149.

[9] S. Sharma et al., PoS Lattice 2013, 164 (2013), arXiv: 1311 . 3943.

[10] O. Kochetkov et al., Talk at Lattice 2014. 